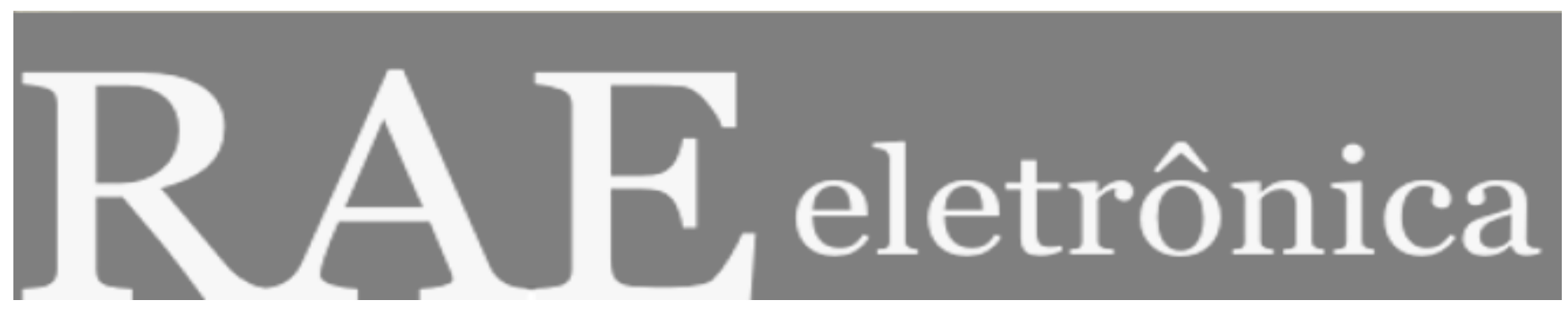

\title{
Tax XML - SISTEMA DE APOIO À COOPERAÇÃO TRIBUTÁRIA INTERNACIONAL
}

Por

\section{Antonio Sergio Seco Ferreira Waldomiro Loyolla}

RAE-eletrônica, Volume 1, Número 1, jan-jun/2002.

http://www.rae.com.br/eletronica/index.cfm?FuseAction=Artigo\&ID=1320\&Secao=INFORMAÇÃO\&Volume=1\&Nu mero $=1 \& A n o=2002$

CCopyright, 2002, RAE-eletrônica. Todos os direitos, inclusive de tradução, são reservados. É permitido citar parte de artigos sem autorização prévia desde que seja identificada a fonte. A reprodução total de artigos é proibida. Os artigos só devem ser usados para uso pessoal e nãocomercial. Em caso de dúvidas, consulte a redação: redacao@rae.com.br.

A RAE-eletrônica é a revista on-line da FGV-EAESP, totalmente aberta e criada com o objetivo de agilizar a veiculação de trabalhos inéditos. Lançada em janeiro de 2002, com perfil acadêmico, é dedicada a professores, pesquisadores e estudantes. Para mais informações consulte o site www.rae.com.br/eletronica.

RAE-eletrônica

ISSN 1676-5648

(C)2002 Editora: Fundação Getulio Vargas - Escola de Administração de Empresas de São Paulo.

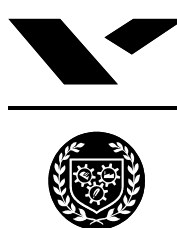




\title{
Tax XML - SISTEMA DE APOIO À COOPERAÇÃO TRIBUTÁRIA INTERNACIONAL
}

\section{Antonio Sergio Seco Ferreira}

Consultor da Unidade de Coordenação de Programas do Ministério da Fazenda. Mestre em Gestão do Conhecimento e Tecnologia da Informação pela Universidade Católica de Brasília.

E-mail: antonio.seco@ieee.org

Endereço: SQN 211 Bloco J Apto. 406. Brasília, DF. CEP 70863-100

Interesses de pesquisa: Governo eletrônico, Lógica nebulosa aplicada, Tecnologias aplicadas à área fiscal.

\section{Waldomiro Loyolla}

Professor do Mestrado em Informática da PUC-Campinas, mestre em Engenharia Elétrica pela USP-EESC e Doutor em Engenharia Elétrica pela UNICAMP-FEEC.

E-mail: loyolla@puc-campinas.edu.br

Endereço: Mestrado em Informática - CEATEC - PUC-Campinas, Rod. D. Pedro I Km 136, Campinas - SP, 13086-900.

Interesses de Pesquisa: Sistemas de Informação,Educação a Distância, Gestão de Sistemas de Informação, Gestão do Conhecimento, Gerenciamento de Fluxo de Trabalho, Teletrabalho.

\section{RESUMO}

O crescimento do comércio eletrônico internacional e a globalização econômica exigem das autoridades tributárias nacionais a ampliação da assistência mútua de modo a evitar ou minimizar tentativas de fraudar, eludir ou sonegar tributos, sendo fundamental, neste contexto, a troca sistemática de informações. Este artigo propõe uma arquitetura de sistema internacional de intercâmbio de informações entre autoridades tributárias, denominado taxXML, baseada em documentos XML não persistentes, Internet e tecnologias padronizadas associadas. São examinados modelos de tratados internacionais e sistemas regionais similares, resultando na proposição de modelo de gestão, operacional e tecnológico. Também é proposto um conjunto de elementos XML que implementam os documentos a serem enviados, os quais foram inicialmente estruturados por meio de grafos orientados e em seguida modelados utilizando o padrão W3C XML Schema. É realizada uma comparação de taxXML com sistemas similares, e são mostrados exemplos de instâncias de documentos taxXML. Finalizando, são identificados trabalhos adicionais que poderiam fortalecer a arquitetura proposta.

\section{PALAVRAS-CHAVE}

Comércio eletrônico, globalização econômica, tributação, intercâmbio de informações, taxXML.

\begin{abstract}
The electronic commerce growth and the economic globalization demand expand of the mutual assistance among the national tax authorities in order to avoid or minimize taxes fraud, elusion or evasion, therefore making the systematic exchange of information a fundamental process in this context. This paper proposes an information interchange international system architecture, named taxXML, which is based on non-persistent XML documents, Internet and associated standard technologies. International treaties models and similar regional systems were examined, resulting on the proposition of a management, operational and technological model. Also is proposed a XML elements set, which implements the documents to be interchanged, initially structured with oriented graphs and subsequently modeled with the standard W3C XML Schema. A comparison is made between taxXML and others similar systems, and instances of taxXML documents are presented as examples. Finally, additional works are identified in order to strengthen the proposed architecture.
\end{abstract}

\section{KEY WORDS}

Electronic commerce, economic globalization, taxation, information interchange, taxXML. 


\section{INTRODUÇÃO}

O crescimento do comércio eletrônico e a globalização econômica representam grandes desafios para as autoridades tributárias, os quais, associados às novas tecnologias da informação, representam também oportunidades de melhorias nos processos de gestão tributária. Um estudo sobre as tecnologias da informação aplicáveis à gestão da tributação do comércio eletrônico pode ser encontrado em [Ferreira,2001]. Uma das melhorias potenciais identificadas está baseada na necessidade de ampliar e generalizar acordos internacionais de assistência entre autoridades tributárias, e fundamentá-los no intercâmbio sistemático de informações tendo por suporte as modernas tecnologias da informação, evitando ou minimizando tentativas de fraudar, eludir ou sonegar tributos em operações comerciais internacionais.

Tal visão é corroborada pela pauta de recentes seminários especializados internacionais, como a "Conferencia Técnica del CIAT - 2001",, na qual participaram autoridades tributarias de todo o mundo além de representantes de organismos internacionais, realizado em outubro de 2001 na Espanha. No programa da Conferencia, é proposta a discussão do tema "Nuevas tecnologías e intercambio de información entre Administraciones", como requisito essencial para uma gestão tributária eficaz.

Uma alternativa para implementar este requisito seria a construção de um sistema internacional de intercâmbio de informações, aberto e facilmente expansível, utilizando a Internet e as tecnologias padronizadas a ela associadas.

Este artigo apresenta uma proposta de arquitetura para este sistema, denominado taxXML, baseado em um vocabulário comum organizado sob a forma de documentos XML (eXtensible Markup Language $)^{\mathrm{ii}}$. As funcionalidades para o sistema são propostas a partir de experiências regionais bem sucedidas e de recomendações de especialistas, resultando em proposições de modelo de gestão, modelo operacional e modelo tecnológico. No modelo tecnológico, são definidas "ações" (requerimentos de serviço) e "reações" (respostas a cada requerimento), implementadas em documentos XML e transportadas em protocolo HTTP (Hyper-Text Transport Protocol). O vocabulário requerido é modelado em documentos XML, utilizando-se o padrão W3C ${ }^{\mathrm{iii}} X M L$ Schema, com o apoio de grafos orientados e técnicas descritivas. Como exemplo, são apresentadas instâncias de documentos taxXML para implementar algumas funcionalidades. Para um melhor entendimento da abordagem proposta, o taxXML é comparado com outros sistemas regionais similares. Finalizando, são discutidos aspectos de segurança e possíveis requisitos futuros para fortalecimento da arquitetura e ampliação do escopo do sistema.

\section{REFERÊNCIAS NACIONAIS E INTERNACIONAIS}

$\mathrm{Na}$ gestão tributária internacional do comércio convencional já era sentida a necessidade de mecanismos de cooperação e intercâmbio de informações entre autoridades tributárias de distintas. Conforme [OECD-MTC,2001], mais de 1500 acordos bilaterais e multilaterais, entre países ou grupos de países com interesses comuns, já foram assinados para promover a assistência mútua na gestão tributária, embora os intercâmbios de informações sejam definidos caso a caso e poucos estejam baseados em sistemas informatizados. No entanto, a globalização econômica e o enorme crescimento do comércio eletrônico internacional recrudesceram as necessidades no sentido de um intercâmbio de informações padronizado, mais ágil, disponibilizando maior quantidade de informações e suportado por sistemas computadorizados. As formas de intercâmbio de informações conforme mencionadas em [Lozano,2001] são:

Intercâmbio esporádico: autoridade tributária competente formula uma pergunta específica; 
Intercâmbio automático: informações acordadas são enviadas de modo sistemático;

Intercâmbio espontâneo: informações julgadas de interesse para outro Estado são a ele remetidas;

Fiscalizações simultâneas: fiscalizações de empresas são organizadas com a participação de funcionários de distintos Estados.

Modelos de acordos desta natureza foram desenvolvidos pela OECD (Organização para Cooperação e Desenvolvimento Econômico) e pela ONU (Organização das Nações Unidas), os quais, embora destinados a informações tributárias específicas, podem ser utilizados para um intercâmbio ampliado de informações. Mais recentemente o CIAT (Centro Interamericano de Administraciones Tributarias ${ }^{\text {iv }}$ ) divulgou o "Modelo de Acuerdo para Intercambio de Informaciones Tributarias del CIAT", proposto para ser adotado entre os países membros da organização (todos os países das Américas). Neste modelo, estão definidos procedimentos para a solicitação de intercâmbio de informações e a realização de fiscalizações específicas e simultâneas. A identificação das informações a serem trocadas e os detalhes dos procedimentos de fiscalização não são parte do modelo, ficando por conta de negociações entre as partes envolvidas.

No entanto, dois acordos regionais são dignos de destaque, por estabelecerem compromissos de entrega periódica de determinadas informações plenamente identificadas, e serem formalmente baseados em sistemas informatizados e redes de comunicações.

\section{VIES (VAT Information Exchange System)}

O sistema VIES foi adotado pelos países da Comunidade Européia, em 1993, para o intercâmbio de informações sobre o imposto sobre o consumo (Value Added Tax ou Imposto sobre o Valor Agregado). Com o fim das barreiras físicas de fronteira entre aqueles países, era necessário um sistema que continuasse provendo informações sobre compras e vendas de bens e serviços, já que o tributo sobre o consumo continuava sendo totalmente absorvido pelo país de destino ou consumo do bem ou serviço [Neves\&Rodrigues,1997].

Este sistema foi baseado em uma rede de comunicações de dados baseada no padrão CCITT X.25, construída e operada por terceiros, que conecta as administrações tributárias dos países membros da Comunidade. Sobre esta rede são oferecidos 2 serviços básicos:

Acesso on-line a alguns dados cadastrais de empresas registradas nos países membros;

Trocas periódicas de lotes de notas fiscais de operações de comércio realizadas entre cada par de países.

A solicitação de informações ou intervenções que não constam do sistema são negociadas por outros meios (FAX, e-mail, etc.), não havendo um idioma padronizado ou mesmo recomendado.

A operação da rede e do sistema é realizada centralmente, na sede da Comunidade em Bruxelas, por empresa terceirizada que não tem acesso aos dados que trafegam na rede. A gestão administrativa do sistema é realizada por um Comitê de Gestão, formado por representantes das administrações tributárias participantes, que se reúne periodicamente em Bruxelas.

\section{SINTEGRA (Sistema Integrado de Intercâmbio de Informações sobre o ICMS)}

O maior tributo no Brasil em volume de arrecadação, ICMS (Imposto sobre Circulação de Mercadorias e Serviços), é administrado pelos Estados brasileiros, e suas características exigem 
uma assistência mútua constante entre as administrações tributárias estaduais, para a gestão do comércio interestadual. O fórum para discussão dos temas relativos ao tributo é o CONFAZ (Conselho Nacional de Política Fazendária), formado pelo Ministro da Fazenda e Secretários de Fazenda das 27 Unidades da Federação (Estados e Distrito Federal). Desde 1995 existia a disposição de que os contribuintes que realizassem operações de comércio interestadual deveriam enviar disquetes contendo determinadas informações às administrações tributárias estaduais correspondentes (Convênio ICMS 57/95 $5^{\mathrm{V}}$ ). Esta disposição era seguida por poucos contribuintes, tampouco as Secretarias de Fazenda exigiam seu cumprimento, principalmente por não terem capacidade administrativa e operacional para tratar as informações solicitadas.

Em 1997 foi decidida a criação do SINTEGRA, destinado a automatizar a assistência entre as administrações tributárias estaduais brasileiras. O SINTEGRA, conceitualmente, se moldou no VIES. Entretanto, ampliou os serviços oferecidos entre administrações tributárias, ofereceu novos serviços aos contribuintes e elevou o nível da tecnologia utilizada [Botaro,1999].

Este sistema está baseado em uma Intranet baseada em VPN (Virtual Private Network), montada sobre uma rede governamental de alcance nacional, mantendo a autonomia constitucional das Unidades da Federação. Em termos computacionais, esta autonomia é representada pela inexistência de uma base de dados central, mantendo cada Secretaria Estadual de Fazenda suas bases de dados e intercambiando dados com as demais por meio da rede. Os serviços oferecidos entre administrações tributárias estaduais, através da Intranet, são:

Acesso on-line a dados cadastrais de empresas registradas nos Estados e Distrito Federal;

Trocas periódicas de lotes de notas fiscais de operações de comércio realizadas entre cada par de Estados;

Sistema padronizado para solicitação de ações fiscais, incluindo o acompanhamento da sua realização;

Sistema padronizado de confrontação das informações intercambiadas, para detecção de indícios de fraudes;

Aos contribuintes são oferecidos, por meio da Internet, a possibilidade de consultarem alguns dados cadastrais de empresas registradas em cada uma das 27 Unidades da Federação, verificar e recuperar algoritmos de geração de números cadastrais, e acesso à legislação específica. $\mathrm{O}$ site público para acesso a estes serviços e obtenção de informações adicionais sobre o sistema é http://www.sintegra.gov.br .

A gestão do sistema é realizada por um Grupo de Trabalho formado por representantes dos Estados participantes, no âmbito do CONFAZ.

Ambos sistemas apresentados são exemplos de cooperação bem sucedida entre administrações tributárias. $\mathrm{O}$ advento do comércio eletrônico e a globalização exacerbam esta necessidade de assistência mútua, agora em nível internacional.

\section{OBJETIVOS DO SISTEMA taxXML}

O sistema taxXML tem por objetivo principal possibilitar o intercâmbio de informações eficiente e ágil entre autoridades tributárias de diferentes países, servindo de suporte para a implementação de acordos multilaterais de assistência. Para alcançar seu objetivo, está baseado nas seguintes premissas: 
Utilizar a Internet como rede de comunicações;

Utilizar padrões tecnológicos internacionais;

Estar baseado na troca de documentos XML;

Possibilitar expansão modular (inclusão incremental de novos documentos);

\section{FUNCIONALIDADES BÁSICAS}

De modo a suportar as formas básicas de intercâmbio de informações mencionadas anteriormente, foram selecionadas as seguintes funcionalidades a serem implementadas na versão inicial da arquitetura de taxXML:

Consulta on-line a elementos pré-fixados de cadastros de contribuintes nacionais;

Envio de pedido de verificação fiscal (auditoria), podendo serem anexadas notas fiscais e imagens de documentos relevantes;

Acompanhamento de evolução de pedido de verificação fiscal;

Envio de resultado de pedido de verificação fiscal;

Envio de informações não solicitadas (intercâmbio espontâneo), podendo serem anexadas notas fiscais e imagens de documentos relevantes.

Para evitar que o funcionamento de uma autoridade tributária seja emperrada por excesso de solicitações de pedidos de verificação fiscal, nos convênios de assistência a serem celebrados entre autoridades tributárias podem ser especificadas cotas máximas periódicas de pedidos, as quais serão controladas pelo sistema computacional.

Estas funcionalidades deverão ser implementadas com mecanismos que assegurem a confidencialidade e integridade dos dados e a identificação das autoridades tributárias envolvidas.

Uma visão abrangente da arquitetura proposta para o sistema taxXML será apresentada por meio do seu Modelo de Gestão, Modelo Operacional, Modelo Tecnológico e da modelagem do vocabulário dos documentos XML que implementarão as funcionalidades estabelecidas anteriormente.

\section{MODELO DE GESTÃO DO SISTEMA taxXML}

O taxXML, sendo um sistema de intercâmbio de informações entre autoridades tributárias de diferentes países, requer uma estrutura de gestão abrangente, com representatividade de todos os participantes. Tal estrutura de gestão, possivelmente representada por um Comitê Gestor composto por representantes dos países participantes, poderia ser criada no âmbito de um organismo internacional que disporia da infraestrutura organizacional adequada, além da experiência na constituição e suporte de grupos similares. As principais responsabilidades do Comitê Gestor estariam divididas em dois grupos, possivelmente tratadas por equipes diferentes:

\section{Gestão Operacional Internacional:}

Avaliar relatórios operacionais e de cumprimento de normas de uso;

Viabilizar um fórum adequado ao intercâmbio de experiências quanto ao uso do sistema; 
Divulgar estatísticas e informações relevantes às autoridades tributárias participantes.

\section{Adequação e evolução do sistema:}

Divulgar padrões adotados;

Definir modelos de convênios para adesão ao sistema;

Propor mecanismos de suporte financeiro a serem assumidos pelas autoridades tributárias participantes;

Propor políticas de segurança e privacidade a serem adotados pelas autoridades tributárias participantes para proteção das informações tratadas no sistema;

Avaliar propostas de alterações ou ampliações;

Promover a evolução tecnológica.

\section{MODELO OPERACIONAL DO SISTEMA taXXML}

É recomendável que a gestão operacional do taxXML seja realizada por uma unidade operacional especial criada em cada autoridade tributária, por tratar-se de sistema cujo funcionamento necessitará ser coordenado com entidades externas internacionais.

Esta unidade operativa, a qual denominamos UNIDADE DE ENLACE INTERNACIONAL (UEI), seria responsável por:

Manter o sistema em funcionamento, de acordo a padrões pré-especificados;

Analisar as solicitações recebidas e encaminha-las à unidade administrativa local adequada para seu atendimento;

Alimentar o sistema com status de andamento e respostas definitivas de cada pedido recebido; Recolher dados estatísticos e de performance;

Interagir com a equipe informática local para assegurar a operação efetiva do sistema;

Realizar os contatos necessários com as demais UEI para a garantir que o sistema funcione adequadamente.

A Figura 1 representa graficamente o papel operacional das UEI e suas principais interfaces.

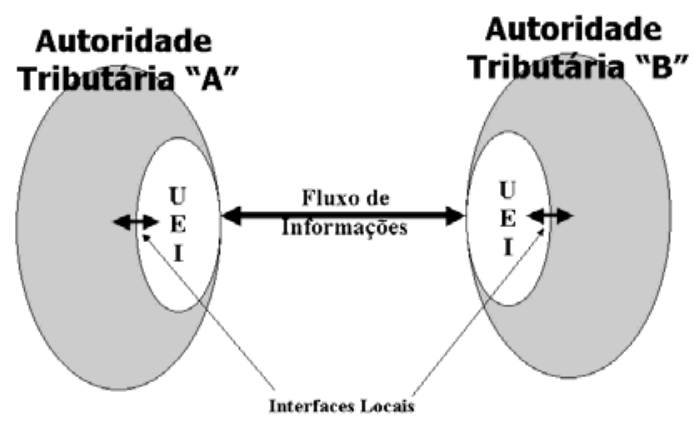




\section{FIGURA 1 - O papel das UEI}

Embora não essencial, seria desejável a integração do taxXML ao sistema informático tributário local, para melhor automatizar funções do sistema que requerem informações produzidas pelos sistemas internos de cada organização.

\section{MODELO TECNOLÓGICO DO SISTEMA taxXML}

A proposta taxXML é baseada no uso da Internet e de tecnologias padronizadas a ela associadas para viabilizar o intercâmbio de informações entre autoridades tributárias de diferentes países, sendo tipicamente um sistema G2G (governo-a-governo). As informações trocadas são documentos XML não persistentes, transportados em protocolo HTTP.

O modelo tecnológico do sistema foi influenciada por modelos desenvolvidos para frameworks de comércio eletrônico B2B fundamentados em documentos XML, tais como cXML [Ariba,2001] e RosettaNet $^{\mathrm{vi}}$.

Outros componentes importantes na concepção do modelo tecnológico são descritos a seguir.

\section{Mecanismo de Intercâmbio}

O intercâmbio de informações entre autoridades tributárias será realizado por transações síncronas de ação/reação, no qual um documento de "ação" (action) enviado a um equipamento servidor taxXML deve ser respondido imediatamente por um documento de "reação" (reaction), o qual avisa ao transmissor da aceitação ou não da "ação" solicitada e, se a "ação" for de resposta imediata, envia também a informação pedida.

O esquema ação/reação está baseado no protocolo de transporte HTTP. Este protocolo, amplamente utilizado na Internet, apresenta maior flexibilidade no tratamento de mensagens síncronas, conforme mostrado na Figura 2, onde A e B representam autoridades tributárias em processo de intercâmbio de informações.

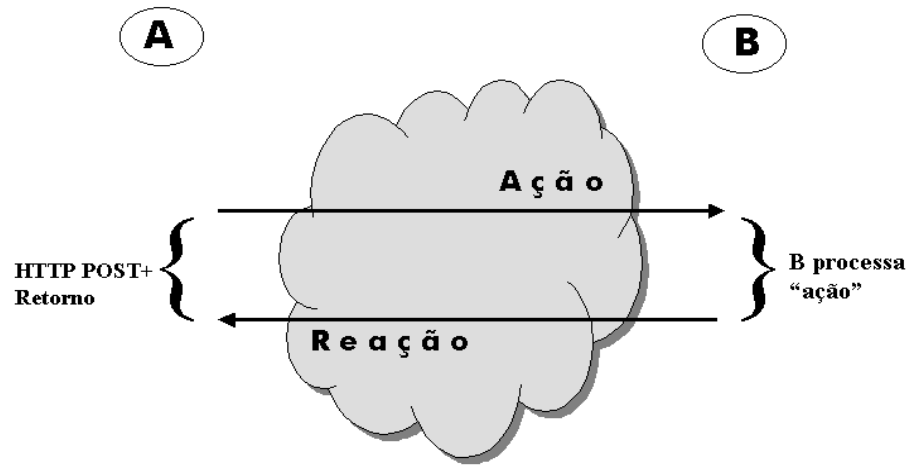

\section{FIGURA 2: Modelo "ação/reação" do taxXML}

\section{Estrutura $n$-Tier}

A implementação do serviço poderá ser realizada com base na arquitetura genérica multicamadas ( $n$-Tier) para serviços na Web, conforme proposta em [Vasudevan,2001], representada na Figura 3. 


\section{FIGURA 3: Proposta de arquitetura - Serviço taxXML}

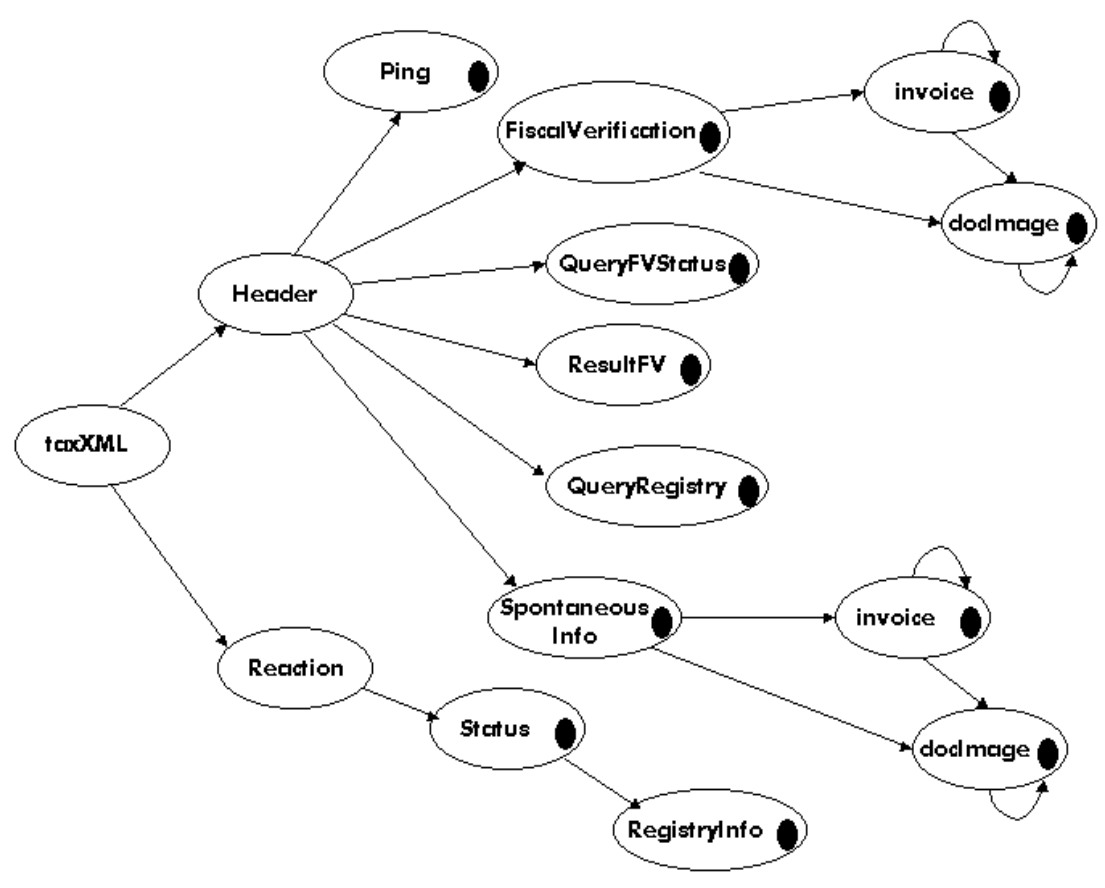

Nesta representação, o "listener" seria uma rotina de uso geral para recepção / envio de mensagens pela Internet; a "fachada" da lógica de negócio representa uma validação preliminar das mensagens recebidas (podendo existir ou não uma reformatação) e seu encaminhamento para tratamento pela "lógica de negócio"; a "lógica de negócio" do taxXML é o suporte ao mecanismo de "ação" e "reação" proposto, e sua interface com o sistema de base de dados utilizado. Sendo o taxXML orientado a mensagens (dados não persistentes), o armazenamento das informações poderá ser realizado em bases de dados relacionais ou nativas XML.

A arquitetura proposta é independente de linguagens de programação e de sistemas de banco de dados específicos. Os equipamentos servidores a serem utilizados e a alocação de camadas em cada um deles depende do contexto local de cada autoridade tributária e da carga esperada sobre o sistema.

\section{MODELAGEM DO VOCABULÁRIO taxXML}

A adoção de um vocabulário comum viabiliza a uniformidade semântica no intercâmbio de informações no seio de uma comunidade, formada no presente caso pelas autoridades tributárias de diferentes países. A modelagem de um vocabulário XML compreende a definição dos elementos utilizados, sua especificação em termos de atributos e tipos de dados, e a estruturação dos relacionamentos viáveis entre eles para a composição de documentos XML.

Deste modo, o vocabulário proposto para taxXML está representado pelos elementos XML que implementam as funcionalidades pretendidas, seus atributos e relacionamentos, organizados sob a forma de documentos XML. As informações intercambiadas estão contidas em um envelope taxXML (elemento raiz), que conduz uma solicitação de "ação" ou a respectiva "reação". Por se tratar de um sistema de uso internacional, os elementos dos documentos (vocabulário) serão nomeados em inglês.

A estruturação adotada para os documentos taxXML assume as seguintes formas gerais: 


\section{Documentos do tipo Ação:}

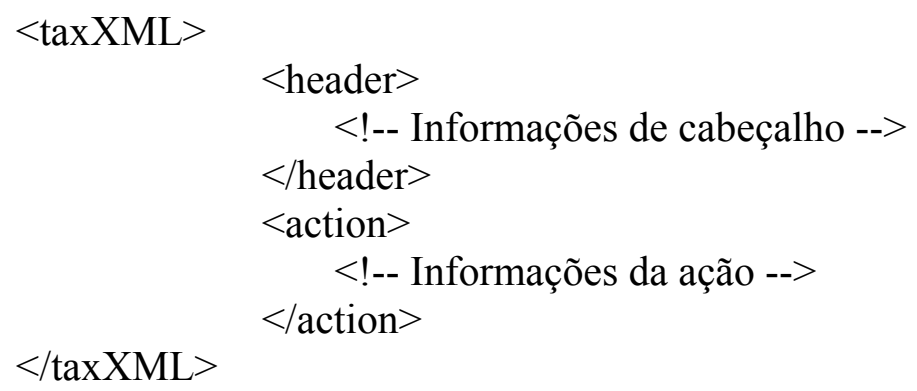

\section{Documentos do tipo Reação:}

$<\operatorname{tax} \mathrm{XML}>$

$<$ reaction $>$

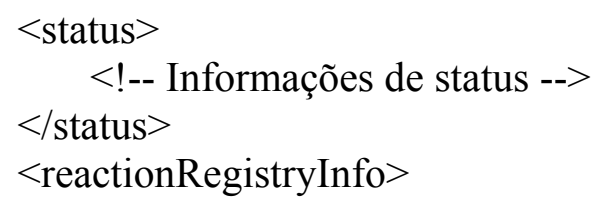

$<$ !-- somente como reação à solicitação de dados cadastrais -->

elementos contendo informações cadastrais ....>

$</$ reaction $>$

$</$ reactionRegistryInfo $>$

$</ \operatorname{tax} X M L>$

Dada a natureza do mecanismo de transporte utilizado, o documento "reação" não necessita de cabeçalho, pois viaja na mesma conexão HTTP.

No processo de modelagem dos documentos taxXML foram inicialmente utilizados mecanismos de grafos orientados e tabelas descritivas. Para a modelagem formal foi adotado o padrão W3C $X M L$ Schema, cuja abordagem é inerente à linguagem XML e, por sua completeza descritiva e formalidade, é mais apropriada para disseminação do vocabulário e para a automatização do processo de validação de documentos.

\section{Elementos de Controle, Ações e Reações Implementadas}

Os elementos de controle definem características gerais associadas a uma ação ou reação. Os principais estão descritos na Tabela I.

\begin{tabular}{|l|l|}
\hline Elemento & Descrição \\
\hline taxXML & $\begin{array}{l}\text { Envelope de transporte das "ações" e "reações"; identifica } \\
\text { univocamente o documento, horário local e idioma utilizado }\end{array}$ \\
\hline header & $\begin{array}{l}\text { Informações sobre as autoridades tributárias de origem e destino em } \\
\text { uma "ação" }\end{array}$ \\
\hline reaction & Engloba outros elementos em uma "reação" \\
\hline status & $\begin{array}{l}\text { Associado sempre a uma "reação", contém informações sobre a } \\
\text { aceitação e/ou o processamento da "ação" que o precedeu }\end{array}$ \\
\hline
\end{tabular}

TABELA I: Elementos de controle propostos para implementação na versão “0” 
As ações propostas para implementação nesta versão " 0 " do sistema foram identificadas a partir das funcionalidades básicas mencionadas anteriormente, e estão sumarizadas na Tabela II, que também inclui as reações esperadas. O nome do elemento XML que implementa a ação ou reação vem imediatamente abaixo, grafadas em itálico.

\begin{tabular}{|c|c|c|c|c|}
\hline Ação & Cód. & Reação & Cód. & Tipo \\
\hline $\begin{array}{l}\text { Solicitação de Verificação Fiscal } \\
\text { (auditoria) } \\
\text { ActionFiscalVerification }\end{array}$ & FV & $\begin{array}{l}\text { Resposta de aceitação ou } \\
\text { não } \\
\text { status }\end{array}$ & $\begin{array}{l}\text { STA } \\
\text { T }\end{array}$ & $\mathrm{N} / \mathrm{D}$ \\
\hline $\begin{array}{l}\text { Solicitação de Status de Andamento } \\
\text { de Verificação Fiscal } \\
\text { ActionQueryFVStatus }\end{array}$ & QFV & $\begin{array}{ll}\text { Resposta com situação de } \\
\text { andamento da Verificação } \\
\text { Fiscal } \\
\text { status }\end{array}$ & $\begin{array}{l}\text { STA } \\
\text { T }\end{array}$ & $\mathrm{N} / \mathrm{O}$ \\
\hline $\begin{array}{l}\text { Envio de Resultado de Verificação } \\
\text { Fiscal } \\
\text { ActionResultFV }\end{array}$ & RFV & $\begin{array}{l}\text { Resposta de aceitação ou } \\
\text { não } \\
\text { status }\end{array}$ & $\begin{array}{l}\text { STA } \\
\text { T }\end{array}$ & $\mathrm{N} / \mathrm{O}$ \\
\hline $\begin{array}{l}\text { Envio Espontâneo de Informações } \\
\text { actionSpontaneousInformation }\end{array}$ & SI & $\begin{array}{l}\text { Resposta de aceitação ou } \\
\text { não } \\
\text { status }\end{array}$ & $\begin{array}{l}\text { STA } \\
\text { T }\end{array}$ & $\mathrm{N} / \mathrm{O}$ \\
\hline $\begin{array}{l}\text { Solicitação de Informações } \\
\text { Cadastrais } \\
\text { actionQueryRegistry }\end{array}$ & QR & $\begin{array}{l}\text { Envio de Informações } \\
\text { Cadastrais } \\
\text { status } \\
\text { reactionRegistryInfo }\end{array}$ & RI & $\mathrm{N} / \mathrm{O}$ \\
\hline $\begin{array}{l}\text { Solicitação de confirmação de } \\
\text { atividade do equipamento servidor } \\
\text { taxXML } \\
\text { actionPing }\end{array}$ & PING & $\begin{array}{l}\text { Resposta com código de } \\
\text { atividade }\end{array}$ & $\begin{array}{l}\text { STA } \\
\mathrm{T}\end{array}$ & $\mathrm{C} / \mathrm{O}$ \\
\hline
\end{tabular}

\section{TABELA II: Ações e reações propostas para implementação na versão "0"}

A coluna "tipo" possui o seguinte significado:

"N" identifica ações relacionadas com o negócio, e "C" ações de controle;

"D” identifica ações que, uma vez aceitas, serão tratadas em tempo diferido, por procedimentos técnicos e administrativos, e "O” identifica ações que deverão ser respondidas on-line, de modo definitivo.

Dois elementos adicionais podem ser utilizados opcionalmente pelas ações actionFiscalVerification e actionSpontaneousInformation, com o propósito de fornecer informações que complementem ou aclarem a ação solicitada:

invoice, que representa as informações contidas em uma nota fiscal. Para os elementos que constituem uma nota fiscal, foi adotada uma adaptação do modelo padrão proposto no âmbito da OECD [OECD-PDA,2000];

docImage, que oferece a possibilidade da anexação da imagens digitais de documentos de interesse para a análise da ação proposta. 


\section{Sintaxe de Acoplamento dos Elementos XML}

A sintaxe de acoplamento define as possibilidades de relacionamentos entre os principais elementos XML definidos, de modo a constituir uma documento taxXML válido, podendo ser representada graficamente por meio de um "grafo orientado" (Figura 4). Neste grafo, os elementos principais constituintes de taxXML são representados pelos nós, e as arestas indicam os percursos permitidos. Os pontos negros no interior dos nós indicam possibilidade de finalizar um percurso.

FIGURA 4: Grafo orientado representando a formação de um documento taxXML.

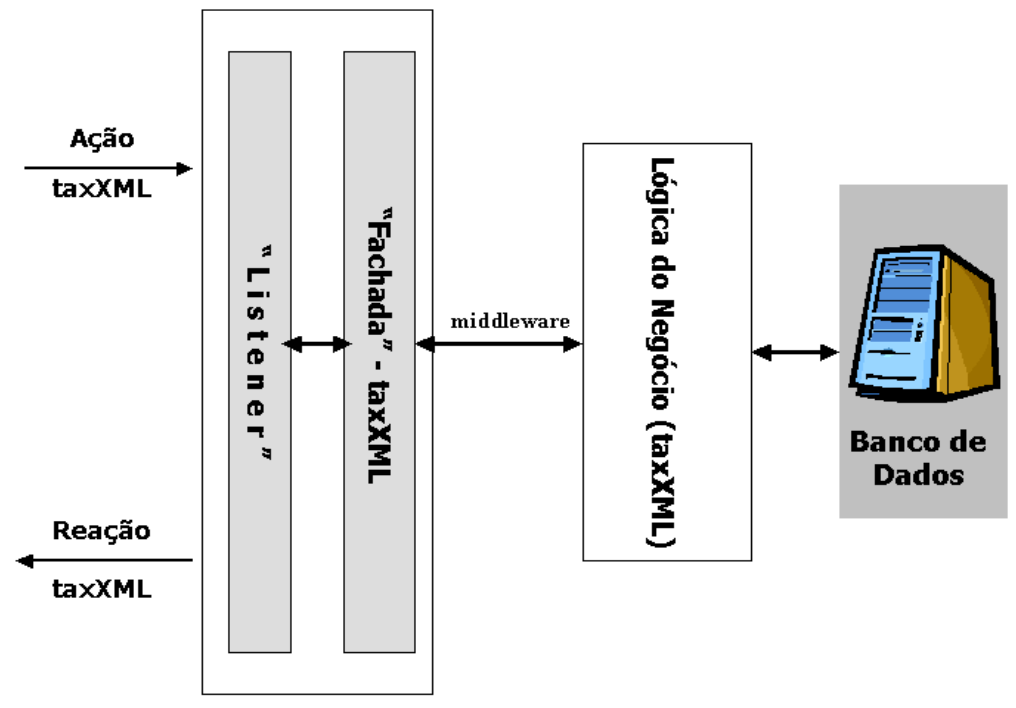

Um percurso se origina sempre no nó taxXML e, a cada nó visitado, o elemento representado é agregado ao documento em formação. Percursos degenerados (nós com conexão a eles mesmos) significam que o elemento pode ser incluído no documento várias vezes.

A seguir, são mostrados alguns exemplos de estruturação de elementos taxXML, originados por percursos no grafo da Figura 4:

taxXML / Header / SpontaneousInformation / invoice / invoice.

taxXML / Header / ResultFV.

taxXML / Header / FiscalVerification / invoice / docImage.

taxXML / Reaction / Status.

Esta abordagem não é suficiente para a criação de um documento válido, porém auxilia na visualização das alternativas possíveis durante o processo de concepção.

\section{Modelagem com XML Schema}

A partir do grafo orientado, cada elemento de taxXML foi detalhado utilizando-se uma abordagem tabular descritiva, que facilita o entendimento inicial. Em seguida, foi realizada uma modelagem formal destes elementos com base em XML Schema.

O XML Schema é um padrão de modelagem de documentos XML em desenvolvimento pelo W3C, que permite definir a sintaxe e a semântica dos documentos. A versão atual, de 2 de maio de $2001^{\text {vii }}$, está categorizada como "recomendação". 
Os tipos de dados utilizados nesta modelagem tomaram por base os seguintes tipos primitivos e derivados:

string - conjunto de tamanho finito de caracteres.

decimal - número decimal de precisão arbitrária, com uma parte inteira e outra fracionária.

integer - derivado do tipo decimal pela fixação da parte fracionária em zero.

date - data no calendário gregoriano no formato AAAA-MM-DD.

dateTime - representa um instante específico do tempo (data e hora), no formato AAAA-MMDDThh:mm:ss, onde T é o separador de data/hora.

anyURI - Uniform Resource Identifier Reference (inclui URL).

language - código de linguagem (idioma), de acordo com a RFC 1766 - compatível com a norma ISO 639-1.

Foram também adotadas as seguintes normas da ISO (International Organization for Standardization):

ISO 3166 Código de País (2 caracteres)

ISO 4217 Código de Moeda (3 caracteres)

ISO 639-1 Código de idioma (2 caracteres)

Para apoiar a especificação do taxXML por meio de XML Schema e a validação de alguns documentos de teste, foram utilizadas as seguintes ferramentas:

XSV versão 7/7/2001 (modo caractere, freeware) - valida uma instância de documento XML utilizando seu modelo em XML Schema;

XMLSPY versão 4 beta $2-26$ de julho de 2001 (modo gráfico, versão free para testes) - edita modelos e instâncias de documentos XML, realizando também validações.

O esquema e os documentos de teste foram construídos utilizando o processador de texto NOTEPAD, do Windows, e o editor do XMLSPY. Também foi utilizada a funcionalidade gráfica da ferramenta XMLSPY para gerar uma representação do esquema de taxXML, posteriormente a sua codificação em XML Schema, com os principais elementos presentes, mostrada na Figura 5.

Naquela figura, o símbolo "+" na extremidade de uma caixa representa um elemento complexo (elemento composto por elementos adicionais).

\section{FIGURA 5: Representação gráfica dos principais elementos - taxXML (XMLSPY)}

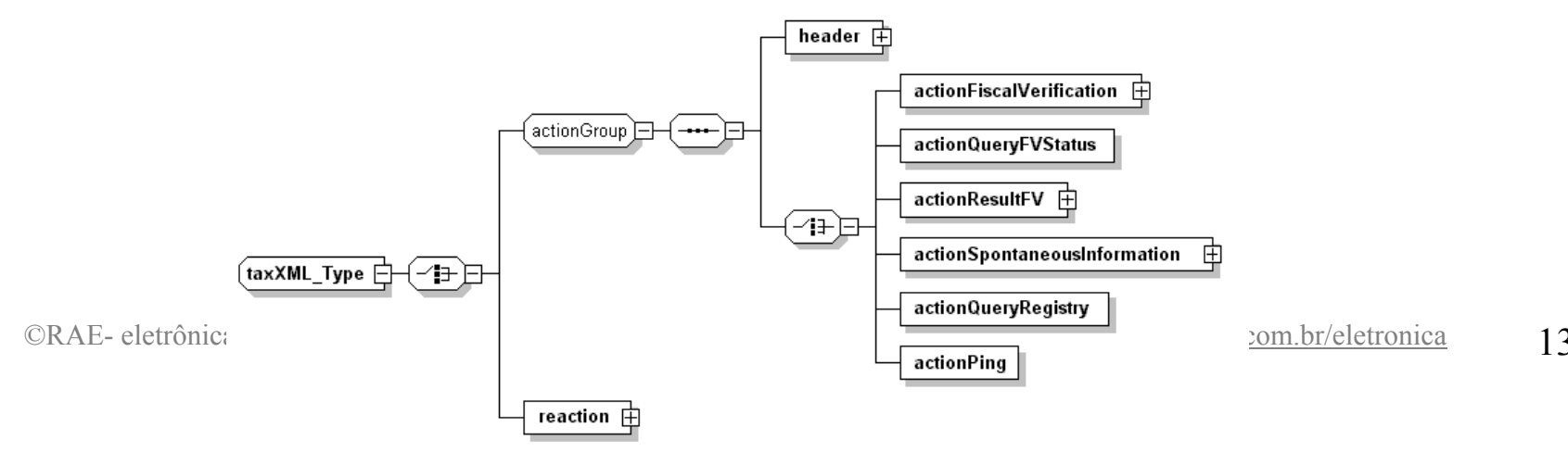


Esta representação, obtida após a construção e validação do esquema, corrobora o diagrama desenvolvido no processo inicial de concepção da estrutura dos documentos taxXML, baseado em grafos orientados e apresentado na Figura 4.

Por tratar-se de uma especificação extensa, na Figura 6 são mostradas apenas para efeito de demonstração as linhas iniciais da modelagem de taxXML em XML Schema. A modelagem completa está disponível no seguinte endereço eletrônico:

http://www.esaf.fazenda.gov.br/cst/arquivos/taxXML-vocabulario-v0.pdf

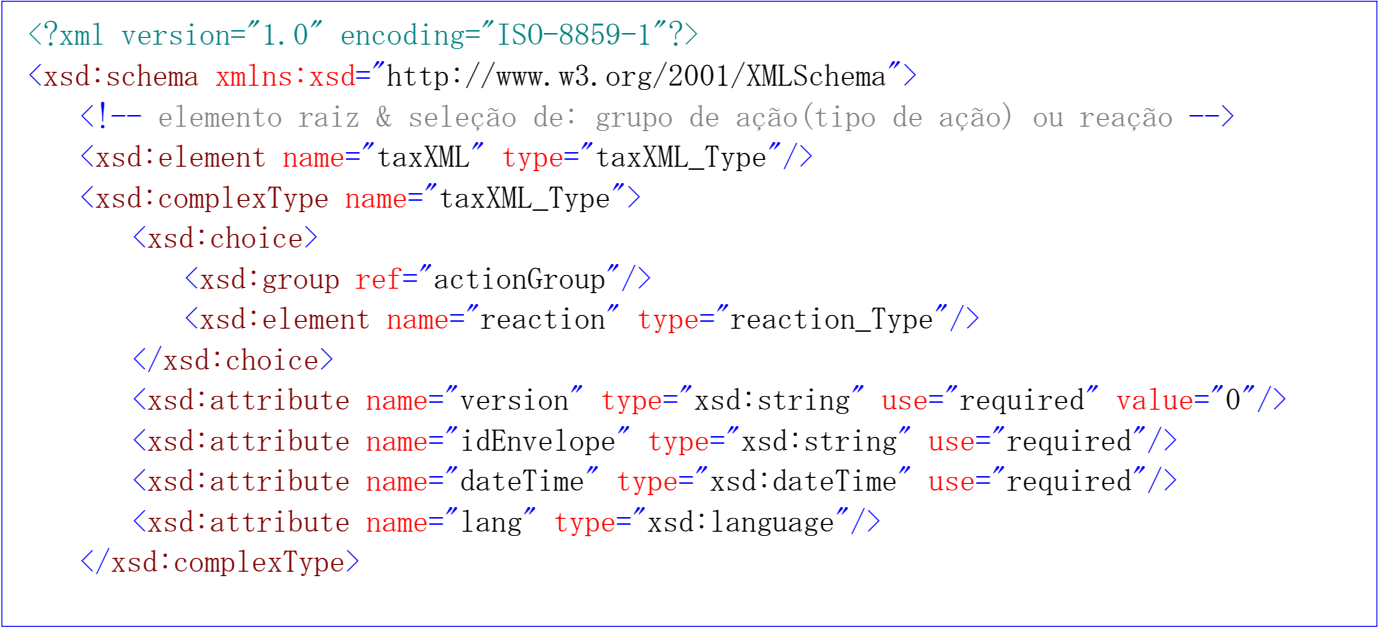

FIGURA 6: Inicio da especificação taxXML em XML Schema

\section{EXEMPLOS DE INTERCAMBIO DE INFORMAÇÕES UTILIZANDO taxXML}

A seguir é apresentado um exemplo de implementação de funcionalidade de "ação" e da respectiva "reação".

Consulta On-line a Elementos do Cadastro de Contribuintes

Esta funcionalidade permite que a autoridade tributária de um país consulte alguns dados do cadastro tributário de outro país. É a única funcionalidade que exige resposta definitiva on-line, sendo implementada por meio da ação actionQueryRegistry e da reação reactionRegistryInfo, representadas esquematicamente na Figura 7, onde A é a autoridade tributária solicitante (geradora da "ação") e B é a autoridade tributária solicitada (geradora da "reação").

FIGURA 7: Representação gráfica de um intercâmbio de documentos taxXML

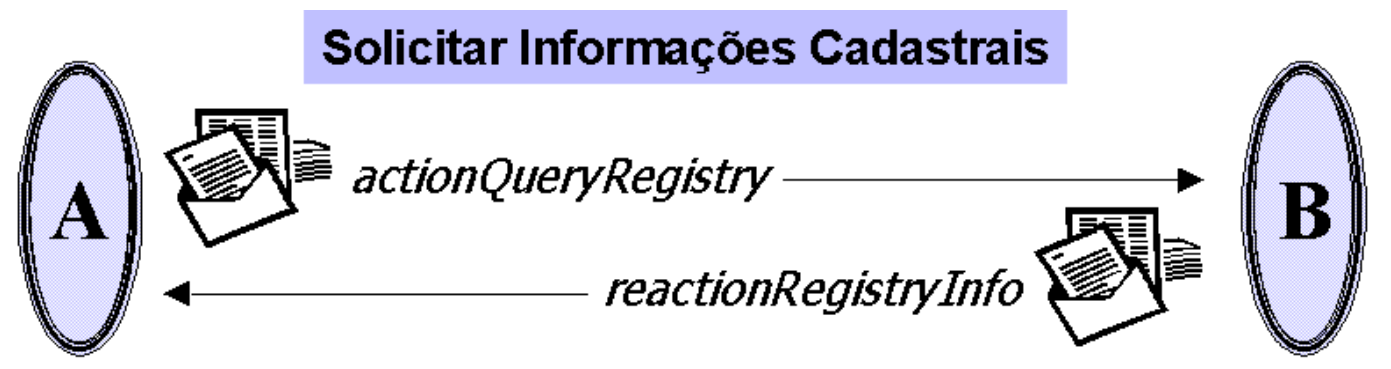


Exemplos de documentos enviados em cada fase são mostrados a seguir, já devidamente validados por meio do XML Schema desenvolvido para o taxXML:

\section{INSTÂNCIA DE DOCUMENTO taxXML para SOLICITAÇÃO DE INFORMAÇÕES CADASTRAIS (actionQueryRegistry)}

$<$ ? $\mathrm{xml}$ version="1.0" encoding="ISO-8859-1"?>

$<$ !-- edited with XML Spy v4.0 beta 2 build Jul 262001 (http://www.xmlspy.com) by Ferreira (mf01) -->

$<!---->$

<!-- EXEMPLO DE DOCUMENTO (SOLICITAÇÃO DE INFORMAÇÕES CADASTRAIS)

actionQueryRegistry -->

$<$ !-- unica ação que requer resultado on-line -->

$<!---->$

$<$ taxXML version="0" idEnvelope="1234455@TUCUNARE" dateTime="2001-0912T13:20:00.000" lang="pt"

xmlns:xsi="http://www.w3.org/2001/XMLSchema-instance"

xsi:noNamespaceSchemaLocation="C: $\mid$ schemas $\$ \#schema-taxXML-v0.xsd">

$<$ header $>$

$<$ from $>$

$<$ originTaxAuthority $>\mathrm{SRF}<$ /originTaxAuthority $>$

$<$ originCountry $>\mathrm{BR}<$ /originCountry $>$

$<$ originContact $>$ João da Silva - tel. $55614122000<$ /originContact $>$

$</$ from $>$

$<$ to $>$

$<$ destinationTaxAuthority $>$ AFIP $</$ destinationTaxAuthority $>$

$<$ destinationCountry $>$ AR $</$ destinationCountry $>$ $</$ to $>$

$</$ header $>$

$</ \operatorname{tax} X M L>$

$<$ actionQueryRegistry taxId="6478916798700"/>

INSTÂNCIA DE DOCUMENTO taxXML DE RESPOSTA A UM A SOLICITAÇÃO DE DADOS CADASTRAIS (reactionRegistryInfo)

$<$ ? $\mathrm{xml}$ version=" 1.0 " encoding="ISO-8859-1"?>

$<$ !-- edited with XML Spy v4.0 beta 2 build Jul 262001 (http://www.xmlspy.com) by Ferreira (mf01) -->

$<!---->$

<!-- EXEMPLO DE DOCUMENTO (RESPOSTA A UMA AÇÃO "actionQueryRegistry")

reaction -->

$<$ !-- -->

$<$ taxXML version="0" idEnvelope="9276500631@SANMARTIN1" dateTime="2001-0912T13:20:35" lang="es"

xmlns:xsi="http://www.w3.org/2001/XMLSchema-instance"

xsi:noNamespaceSchemaLocation="C: $\backslash$ schemas\\#schema-taxXML-v0.xsd">

$<$ reaction $>$

$$
<\text { status code="200" description="OK" }>\text { texto opcional }</ \text { status }>
$$


$<$ reactionRegistryInfo $>$ Antonio Sergio Seco Ferreira - Waldomiro Loyolla

$<\operatorname{tax} I d>6478916728700</ \operatorname{tax} I d>$

$<$ name $>$ Vinicola Santa Juliana $</$ name $>$

$<$ address $1>$ Haciendas Santa Juliana $</$ address $1>$

$<$ city $>$ Mendoza $</$ city $>$

$<$ state $>$ Mendoza $</$ state $>$

$<$ country $>$ AR $<$ country $>$

$<$ status $>1</$ status $>$

$<$ statusDate $>1999-11-30</$ statusDate $>$

$<$ activityDescription $>$ producción de vinos finos $<$ /activityDescription $>$

$</$ reaction $>$

$</$ reactionRegistryInfo $>$

$</ \operatorname{tax} X M L>$

\section{COMPARAÇÃO COM SISTEMAS SIMILARES}

Dois sistemas de intercâmbio de informações entre autoridades tributárias foram examinados no desenvolvimento deste trabalho, VIES e SINTEGRA, descritos anteriormente. A comparação entre estes sistemas e o taxXML, em termos de arquitetura e de suporte a requisitos para um novo ambiente de comércio eletrônico internacional e de globalização econômica, é relevante para um melhor entendimento das distintas abordagens e das potencialidades de cada um (Tabela III).

\begin{tabular}{|l|l|l|l|}
\hline $\begin{array}{l}\text { Formato } \\
\text { intercâmbio }\end{array} \quad$ VIES & Proprietário & Proprietário & XML \\
\hline $\begin{array}{l}\text { Adaptabilidade do } \\
\text { vocabulário }\end{array}$ & Baixa & Baixa & Alta \\
\hline $\begin{array}{l}\text { Tecnologia de } \\
\text { suporte }\end{array}$ & Proprietária & Aberta & Aberta \\
\hline $\begin{array}{l}\text { Cobertura tributária } \\
\text { IVA - Tributo sobre } \\
\text { o consumo }\end{array}$ & $\begin{array}{l}\text { ICMS - Tributo } \\
\text { sobre o consumo }\end{array}$ & Tributos em geral \\
\hline $\begin{array}{l}\text { Comunidade alvo } \\
\text { União Européia }\end{array}$ & $\begin{array}{l}\text { Estados brasileiros } \\
\text { Européia da União }\end{array}$ & COTEPE( $\left(^{*}\right)$ & $\begin{array}{l}\text { Internacional } \\
\text { internacional } \\
\text { (proposta) }\end{array}$ \\
\hline $\begin{array}{l}\text { Integração como } \\
\text { sistemas locais }\end{array}$ & $\begin{array}{l}\text { Responsabilidade de } \\
\text { cada autoridade } \\
\text { tributária }\end{array}$ & $\begin{array}{l}\text { Integração parcial } \\
\text { Responsabilidade de } \\
\text { cada } \\
\text { tributária }\end{array}$ \\
\hline $\begin{array}{l}\text { Estágio } \\
\text { desenvolvimento }\end{array}$ & $\begin{array}{l}\text { Implantado } \\
\text { (1993) }\end{array}$ & $\begin{array}{l}\text { Em implantação } \\
\text { piloto } \\
\text { (final previsto: } \\
\text { 2002) }\end{array}$ & Proposta \\
\hline
\end{tabular}

(*) Comissão Técnica Permanente do ICMS / Estados brasileiros

\section{TABELA III - Comparação - VIES, SINTEGRA, taxXML}

Observa-se que a flexibilidade da abordagem tributária e tecnológica utilizada no taxXML oferece vantagens competitivas no ambiente de comércio eletrônico, onde a tônica é a constante mudança de regras e atitudes por parte dos atores (empresários, consumidores, governo). No entanto as arquiteturas dos sistemas VIES e, em menor escala, SINTEGRA já tiveram sua operabilidade comprovada pela prática, enquanto taxXML está em nível de proposta. 
Dada a abrangência internacional pretendida para o sistema taxXML, um acordo bem sucedido sobre a especificação final do sistema e de sua utilização exigirão esforços consideráveis de coordenação entre autoridades tributárias e organismos internacionais.

\section{CONSIDERAÇÕES SOBRE SEGURANÇA}

A segurança no intercâmbio de informações tributárias exige autenticidade das partes envolvidas, confidencialidade, integridade, e não repúdio das informações enviadas. No âmbito do W3C, os estudos para possibilitar a incorporação diretamente no XML de funções de criptografia e assinatura digital, com granularidade em nível de elementos de um documento, ainda estão embrionárias. Um resumo da situação atual destes trabalhos pode ser encontrado em [Reagle,2001]. No entanto, no plano tecnológico, estes requisitos podem ser implementados com a utilização de certificados digitais de identidade para as autoridades tributárias e do protocolo SSL (Secure Socket Layer) no canal de comunicação. Nesta modalidade, todas as mensagens trocadas durante uma sessão são completamente criptografadas, sem distinção de elementos ou campos. Futuramente, com a evolução dos trabalhos do W3C, poder-se-á dispor de maior granularidade.

Adicionalmente, sistemas de firewall são essenciais para proteção contra acessos indevidos. Neste aspecto, o uso do protocolo HTTP facilita a gestão da segurança, pois a alocação de portas de acesso padronizadas não requer aberturas especiais no firewall.

Localmente, há necessidade de mecanismos de controle lógico de acesso aos sistemas e banco de dados, geralmente baseados em sistemas de usuário / senha que autorizam o acesso a determinados módulos do sistemas.

No plano administrativo, é essencial o controle do acesso físico aos locais críticos (salas de equipamentos servidores, operação de rede, etc.) e a existência de procedimentos de segurança amplamente divulgados e praticados.

A disponibilidade do sistema, outro quesito crítico, dependerá dos equipamentos e do software de base contratados, além da qualidade dos aplicativos desenvolvidos e dos procedimentos operacionais implementados.

Vale ressaltar que todo o esquema de segurança deve estar baseado em uma política geral de segurança para as informações (uso, armazenamento e tráfego). As autoridades tributárias, por lidarem normalmente com informações críticas e confidenciais, possuem experiências administrativas e tecnológicas que facilitarão sua adaptação ao taxXML.

\section{COMENTÁRIOS FINAIS}

A proposta taxXML representa um exemplo prático da aplicabilidade de novas tecnologias da informação na gestão tributária, frente aos novos desafios proporcionados pelo crescimento do comércio eletrônico e da globalização econômica. Para se tornar efetiva, é necessária sua discussão em um fórum mais amplo, com a participação de autoridades tributárias e organismos internacionais, possibilitando a validação de aspectos críticos, como o modelo de gestão e a suficiência dos serviços propostos.

Algumas pesquisas adicionais são necessárias para fortalecimento do arquitetura apresentada. A primeira refere-se à tendência de, futuramente, serem estabelecidos acordos para o intercâmbio massivo de documentos tributários. Para suportar esta facilidade, devem ser analisados outros mecanismos de transporte mais eficientes, além do HTTP proposto nesta versão.

Uma segunda linha refere-se à transformação padronizada dos documentos XML definidos para outros formatos, ampliando sua utilização e aplicações práticas. Neste sentido, destacam-se a transformação para apresentação dos documentos, principalmente em navegadores de Internet, e a exportação destes dados para uso em sistemas legados. Ferramentas baseadas no padrão W3C XSLT (XML Stylesheet Language Transformation) $)^{\text {vii }}$ apresentam-se como candidatas potenciais para esta tarefa. 
Finalmente, conforme já sugerido em [Carlson,2001], a modelagem dos documentos taxXML por meio de UML (Unified Modeling Language) poderia ser futuramente avaliada como forma de obtenção maior rigor nas especificações, e de integração a um ambiente unificado de modelagem.

\section{Artigo recebido em 06.12.2001. Aprovado em 23.03.2001}

\section{REFERÊNCIAS BIBLIOGRÁFICAS}

[Ariba,2001] Ariba, Inc. cXML User's Guide. Version 1.2. February, 2001.

[Botaro,1999] Botaro, Renato. Modernização da Área Fazendária dos Governos Estaduais. Anais do $27^{\circ}$. SECOP. João Pessoa, Paraíba. Novembro de 1999.

[Carlson,2001] Carlson, David. Modeling XML Applications with UML. Upper Saddle River, NJ: Addison-Wesley, 2001.

[Ferreira,2001] Ferreira, Antonio Seco. Tecnologias de Suporte à Tributação do Comércio Eletrônico. Dissertação de Mestrado. Universidade Católica de Brasília. Novembro de 2001.

[Lozano,2001] Lozano, Francisco. La Cooperación Administrativa Internacional para Combatir la Evasión Tributaria y el Contrabando. Anais da 35a. Asambléa General del CIAT. Santiago, Chile. Abril de 2001.

[Neves\&Rodrigues,1997] Neves, Antonio \& Rodrigues, Ema. Sistema VIES. Anais do Seminário Internacional Gestão da Tributação sobre o Consumo nos Estados Brasileiros e na União Européia. Palmas, Tocantins. Maio de 1997.

[OECD-MTC,2001] OECD - Model Tax Convention. Sumário. Disponível em:

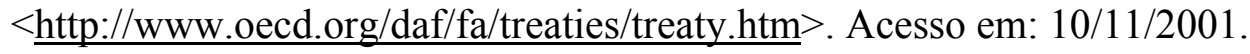

[OECD-PDA,2000] OECD - Technical Advisory Group. Professional Data Assessment Report. December, 2000. Disponível em: $<$ http://www.oecd.org/daf/fa/e com/public release.htm $>$. Acesso em: 23/03/2001.

[Reagle,2000] Reagle Jr., Joseph. The Status and Design of XML Signatures and Encryption. Anais do XML Europe 2001. Berlim, Alemanha. Maio de 2001.

[Vasudevan,2001] Vasudevan, Venu. A Web Service Primer. XML.com, abril de 2001. Disponível em: <www.xml.com/pub/a/2001/04/04/webservices/index.html $>$. Acesso em: 20/07/2001.

\section{Notas:}

1 Centro Interamericano de Administraciones Tributarias - Programa da Conferência disponível em http://www.ciat2001.aeat.es/restemarios/home.html

ii Descrição e especificações em http://www.w3.org/xml

iii World Wide Web Consortium - http://www.w3.org

iv http://www.ciat.org

v $\quad$ http://www.confaz.fazenda.gov.br

vi http://www.rosettanet.org

vii http://www.w3.org/TR/2001/REC-xmlschema-2-20010502/

viii Maiores informações em http://www.w3.org/TR/xslt 